\title{
Quiescent Magnetar Emission: Resonant Compton Upscattering
}

\author{
Matthew G. Baring \\ Rice University, Department of Physics and Astronomy, MS-108, P.O. \\ Box 1892, Houston, TX 77251-1892, USA
}

\begin{abstract}
A principal candidate for quiescent non-thermal gamma-ray emission from magnetars is resonant inverse Compton scattering in the strong fields of their magnetospheres. This paper outlines expectations for such emission, formed from non-thermal electrons accelerated in a pulsar-like polar cap potential upscattering thermal X-rays from the hot stellar surface. The resultant spectra are found to be strikingly flat, with fluxes and strong pulsation that could be detectable by $G L A S T$.
\end{abstract}

\section{Introduction}

The quiescent, non-thermal X-ray emission of soft gamma repeaters (SGRs) and anomalous X-ray pulsars (AXPs) is observed at levels that are very intense for neutron stars. It is quite possible that SGRs and AXPs emit in a broad-band, pulsar-like mode, even if the power is not of a rotational origin. A principal candidate for such emission is inverse Compton scattering, tapping abundant surface X-rays (inferred surface temperatures for AXPs and SGRs are somewhat greater than for canonical pulsars; see Perna et al. 2001) as targets for energetic electrons accelerated in either polar cap or outer gap potentials. Recently, Cheng \& Zhang (2001) proposed a model for significant gamma-ray emission from such magnetars, in the context of the outer gap scenario.

Here, the possibility of magnetar gamma-ray emission from polar cap models is explored. In the strong fields of their magnetospheres, the scattering is enhanced by the cyclotron resonance, thereby rendering polar cap models even more promising for generating hard gamma-rays that are potentially detectable by the upcoming Gamma-Ray Large Area Space Telescope (GLAST) mission. The present scenario is similar to previous pulsar (e.g., Sturner, Dermer \& Michel 1995; Daugherty \& Harding 1996) and old gamma-ray burst (Dermer 1990; Baring 1994) models. Primary electrons with Lorentz factors up to some maximum $\gamma_{\max }$ cool in collisions with the X-ray photons, in the process generating a broad spectrum extending into the gamma-ray band.

This paper outlines expectations for such emission spectra, specifically incorporating relativistic cross sections at supercritical fields that are germane to resonant Compton scattering in the magnetar regime. This investigation will focus on a simple one-zone, uniformly-magnetized region as a preparation for real geometry down the line, where the complexity of pair cascading will be explored. It is found that a very flat soft gamma-ray continuum results, with 
spectral cutoffs that depend strongly on the observational perspective to the field. Spectra differ significantly from those produced in outer gap models. Estimates of the anticipated flux indicate that GLAST may well detect magnetars in the $100 \mathrm{MeV}-1 \mathrm{GeV}$ band if they emit efficiently like gamma-ray pulsars.

\section{Resonant Compton Upscattering}

In strong fields the cross section for Compton scattering is resonant at the cyclotron energy and a series of higher harmonics (e.g., see Daugherty \& Harding 1986), effectively increasing the magnitude of the process over the Thomson cross section $\sigma_{\mathrm{T}}$ by of the order of $1 / \alpha_{\mathrm{f}} B$, where $\alpha_{\mathrm{f}}$ is the fine structure constant. Here, as throughout the paper, magnetic fields are written in units of $B_{\mathrm{cr}}=m_{e}^{2} c^{3} /(e \hbar)=4.413 \times 10^{13}$ gauss, the quantum critical field strength. In the non-relativistic, Thomson regime (e.g., see Herold 1979), only the fundamental resonance is retained.

The dominance by the resonance leads to an effective kinematic coupling between the energies $\varepsilon_{\gamma} m_{e} c^{2}$ and $\gamma m_{e} c^{2}$ of colliding photons and electrons, respectively, and the angle of the initial photon $\theta_{\gamma}$ to the magnetic field lines: the cyclotron fundamental is sampled when $\gamma \varepsilon_{\gamma}\left(1-\cos \theta_{\gamma}\right)=B$. The simplicity of this coupling automatically implies that integration over an angular distribution of incoming photons results in a flat-topped emission spectrum for Compton upscattering in strong magnetic fields. This characteristic is well-documented in the literature (e.g., see Dermer 1990; Baring 1994 for old gamma-ray burst scenarios, and Sturner et al. 1995 for pulsar contexts), specifically for collisions between ultrarelativistic electrons and thermal X-rays emanating from a neutron star surface.

For supercritical magnetar strength fields, the Thomson regime is no longer operable, with relativistic corrections to the Compton cross section becoming required. These have been explored by Gonthier et al. (2000) for cases appropriate to pulsars, namely when photons move parallel to field lines in the electron rest frame. The cross section is suppressed roughly by a Klein-Nishina reduction when $B \gtrsim 1$ so that at $B \sim 100$ it becomes comparable to $\sigma_{\mathrm{T}}$ in the cyclotron resonance, and much smaller for other energies. The analytic developments of Gonthier et al. (2000) for the resonant Compton cross section are employed in the computations of this paper.

The development of emission spectra requires the determination of equilibrium electron/pair distributions. The resonant upscattering of surface X-rays rapidly cools the electrons, resulting in the cessation of acceleration in the polar cap potential gap at $\gamma \lesssim \gamma_{\max } \sim 3 \times 10^{5}-10^{6}$ (e.g., Sturner 1995; Daugherty \& Harding 1996; Harding \& Muslimov 1998). As the primary electrons escape the gap, they continue to cool until the photon density is diluted at altitudes above one stellar radius from the surface, when the scattering kinematics also no longer sample the resonance. The simplest description of such cooling is explored here through standard kinetic equations for development of the electron distribution. The evolution with altitude yields a mean electron distribution (i.e., averaged over the electron path to higher altitudes) that is approximately proportional to the inverse of the cooling rate $\dot{\gamma}_{\mathrm{C}}$. This amounts to a steep distribution (roughly $\propto \gamma^{-4}$ ) for a range in $\gamma$ of generally at least two decades below $\gamma_{\max }$, with a 
very flat distribution (almost independent of $\gamma$ ) at lower energies. There is also a "pile-up" of electrons at mildly-relativistic energies due to the concomitant inefficiency of cooling. Such shapes can be directly inferred from cooling profiles exhibited by Baring (1994) and Sturner (1995), who specialized to the magnetic Thomson limit, and extend at least in general nature to the relativistic domain, where the slopes of the primary electron distribution are modified only slightly.

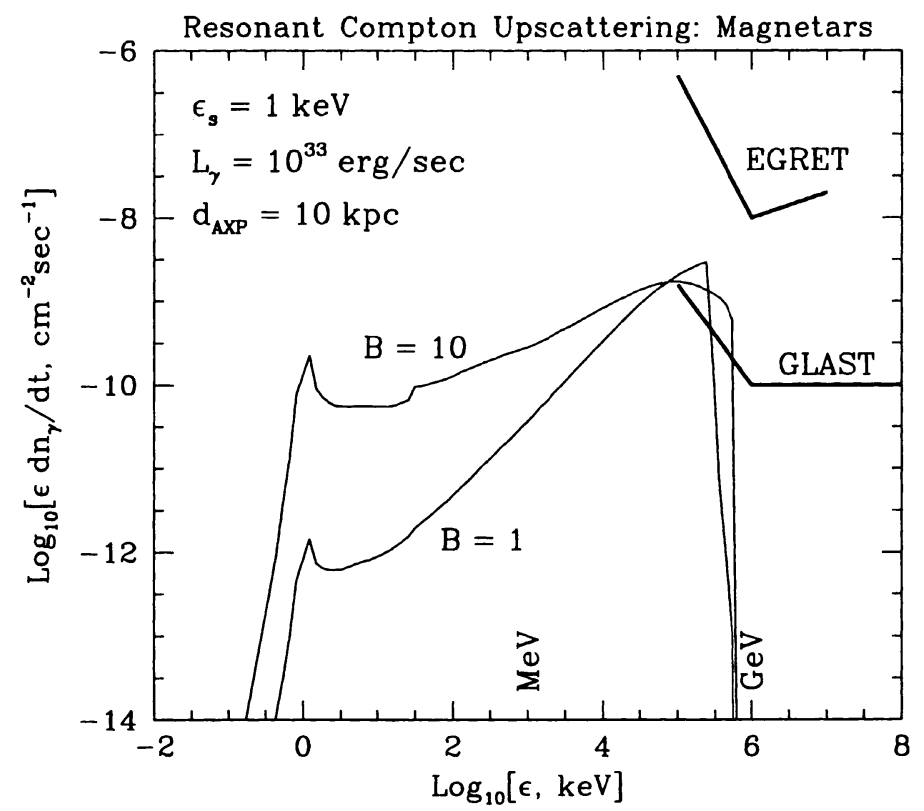

Figure 1. The angle-integrated $F_{\nu}$ spectra for resonant Compton upscattering, for the two cases of $B=1$ and $B=10$, with field strengths $B$ expressed in terms of $B_{\mathrm{cr}}=m_{e}^{2} c^{3} /(e \hbar)=4.413 \times 10^{13}$ gauss. The spectra are normalized for a luminosity of $10^{33} \mathrm{ergs} \mathrm{s}^{-1}$ (see text), and source distance of $10 \mathrm{kpc}$, typical for AXPs and SGRs. An X-ray seed energy of $\varepsilon_{s}=1 \mathrm{keV}$ was used in the models. The EGRET and GLAST flux sensitivities are also depicted.

This Compton-cooled distribution was then imported into standard integrations for the resonant inverse Compton scattering spectrum, which in the Thomson limit were detailed, for example, in Dermer (1990) and Baring (1994). The principal complication incurred by introducing the relativistic cross section is an extra integration as a consequence of the finite energy change of photons in the Klein-Nishina domain; this is handled routinely in the numerical integration routines. Output results of these integrations are exhibited in Figure 1 for two different field strengths. The $B=1$ case is similar in nature to the Thomson regime results of Dermer (1990) and Baring (1994), corresponding to a flat $\varepsilon^{-0}$ distribution. Klein-Nishina effects manifest themselves in the $B=10$ case, that most appropriate to magnetars, as a spectral steepening to around an $\varepsilon^{-2 / 3}$ spectrum. Both cases extend out to photon energies around $1 \mathrm{GeV}$, which reflect the upscattered energy from copious cooling off surface X-rays by 
electrons with $\gamma \sim \gamma_{\max }$. These photons are highly collimated almost along the magnetic field. Some pair creation would be expected near this $\mathrm{GeV}$ cutoff (see the discussion of Gonthier et al. 2000); its exploration is deferred to future work.

The normalizations for the spectra in Figure 1 are established using an obvious observational benchmark. Since a pulsar mode for magnetar gammaray emission is considered here, the correlation between spin-down power $\dot{E}_{\mathrm{SD}}$ and gamma-ray luminosity $L_{\gamma}^{\text {obs }}$ observed by the EGRET experiment on the Compton Gamma-Ray Observatory (CGRO), namely

$$
L_{\gamma}^{\text {obs }} \sim 1.7 \times 10^{16} \sqrt{\dot{E}_{\mathrm{SD}}} \approx 5.3 \times 10^{19} \frac{B_{\text {gauss }}}{P_{\text {sec }}^{2}} \operatorname{ergs~s}^{-1},
$$

can be extrapolated to the magnetar domain. This equates to roughly $10^{32}-10^{33}$ $\mathrm{ergs} \mathrm{s}^{-1}$ for AXPs and SGRs, corresponding approximately to their $\dot{E}_{\mathrm{SD}}$. Note that this luminosity is still two orders of magnitude smaller than the detected quiescent X-ray luminosities, whose power cannot be of rotational origin.

The spectra were normalized to this $L_{\gamma}^{\text {obs }}$ roughly around $1 \mathrm{GeV}$, for a source $10 \mathrm{kpc}$ distant, resulting in predicted fluxes that would be detectable by GLAST, a principal conclusion of this paper. The flat spectra strongly contrast to outer gap predictions of Cheng \& Zhang (2001), which are inherently steeper due to their use of non-magnetic inverse Compton scattering. The polar cap model therefore indicates that EGRET would not have been expected to detect AXPs unless the gamma-ray luminosity was comparable to the X-ray power.

Observe that potential GLAST detections would possess marked phase dependence. The kinematics of resonant scattering yields the strong correlation $\varepsilon_{\gamma} \propto\left(1-\cos \theta_{\gamma}\right)^{-1}$ between the gamma-ray energy $\varepsilon_{\gamma}$ and its angle to the field $\theta_{\gamma}$, translating into much softer spectra at significant angles to the field, i.e., out of the main pulse. Another discriminating feature is the expectation of strong polarization in the gamma-ray signal, though this cannot be probed until future generation missions. It is anticipated that any GLAST detections of AXPs or SGRs in quiescence will have a profound impact on our understanding of these sources, with potential discrimination between outer gap and polar cap models.

\section{References}

Baring, M. G. 1994, in AIP Conf. Proc., Vol. 307, Gamma-Ray Bursts, ed. G. Fishman, (New York: AIP), p. 572

Cheng, K. S., \& Zhang, L. 2001, ApJ, 562, 918

Daugherty, J. K., \& Harding, A. K. 1986, ApJ, 309, 362

- 1996, ApJ, 458, 278

Dermer, C. D. 1990, ApJ, 360, 197

Gonthier, P. L., et al. 2000, ApJ, 540, 907

Harding, A. K., \& Muslimov, A. G. 1998, ApJ, 500, 862

Herold, H. 1979, Phys. Rev. D, 19, 2868

Perna, R., et al. 2001, ApJ, 557, 18

Sturner, S. J. 1995, ApJ, 446, 292

Sturner, S. J., Dermer, C. D., \& Michel, F. C. 1995, ApJ, 445, 736 